\title{
Strong spin-oscillation of small spin-1 condensates caused by an inclined weak magnetic field
}

\author{
Y. Z. He, Z. F. Chen, Z. B. Li, and C. G. Bad* \\ State Key Laboratory of Optoelectronic Materials and Technologies, \\ School of Physics and Engineering, Sun Yat-Sen University, Guangzhou, 510275, P.R. China
}

\begin{abstract}
When a magnetic field is applied along a direction deviated from the quantization $Z$-axis, the conservation of total magnetization holds no more. In this case the inclined field can cause a strong spin-evolution via the linear Zeeman term even the field is as weak as a percentage of $m G$. An approach beyond the mean field theory is proposed to study the evolution of small ${ }^{87} \mathrm{Rb}$ condensates under the weak inclined fields. The time-dependent populations of spin-components are given in analytical forms. The evolution is found to be highly sensitive to the magnitude and direction of the field.
\end{abstract}

PACS numbers: $\quad 03.75 . \mathrm{Mn}, 03.75 . \mathrm{Kk}$

\section{INTRODUCTION}

Since the experimental realization of spinor BoseEinstein condensates [1, 2, 3, 4, [5, 6], the spin-evolution of the condensates has become a hot topic due to its academic interest and potential application. The manipulation of the evolution is a central problem 7, 8, 9, 10, 11, 12, 13, 14, 15, 16]. Usually, one applies an external magnetic field lying along the direction of the axis of quantization $(Z$-axis) $[8,18,19,[20]$. In this way the total magnetization of the condensate is conserved, hence the linear Zeeman term of the field plays no role. However, if the direction of the field deviates from the $Z$ axis, the conservation of the total magnetization will not exist. Accordingly, linear Zeeman term will affect the evolution. Since the linear term is much stronger than the quadratic term, a very weak inclined magnetic field might cause remarkable effect. The dynamic response of the condensate to a transversal magnetic field has been studied by Yi and $\mathrm{Pu}$ 21, 22]. Strong oscillation has been found in the evolution of populations of spin-components. Spin squeezing and macroscopic entanglement have been found in the studies of the ground state structures. Since the direction of the field might affect the dynamic phenomena of condensates sensitively, this topic deserves to be further studied.

The aim of this paper is to study the effect of an inclined magnetic field (lying along an arbitrary $Z^{\prime}$-axis) on the spin-evolution of the condensates of ${ }^{87} \mathrm{Rb}$ atoms. Recently, more attention was paid to the study of small condensates with lower densities because the dissipative process can be reduced, and therefore richer phenomena might be observed 24]. For these small systems the validity of the mean field theory might be limited. Therefore, in this paper, an approach beyond the mean field theory is proposed. It turns out that analytical solutions can be obtained as follows.

\footnotetext{
${ }^{*}$ The corresponding author
}

\section{ONE-BODY SYSTEM}

In order to understand better the effect of the inclined $B$ on many-body systems, we study firstly a simple example, namely, the evolution of a single spin- $1{ }^{87} \mathrm{Rb}$ atom under the field. The initial spin-state of the atom $\chi_{\mu}$ is defined in a $Z$-frame, while $B$ is lying along another axis $Z^{\prime}$. The angle between $Z$ and $Z^{\prime}$ is $\theta$. The Hamiltonian $H=-p \hat{S}_{Z^{\prime}}$, where $p=\gamma B$, and $\gamma=g_{F} \mu_{B}$ being the gyromagnetic ratio. The quadratic Zeeman term is much weaker than the linear term (e.g., if $B=1 m G$, the former is $10^{-7}$ times weaker). Therefore, when $Z^{\prime}$ and $Z$ do not overlap, the former can be neglected.

Due to the inclined magnetic field the spin-state at time $t$ becomes

$$
\xi_{\mu}(t)=e^{-i H t / \hbar} \chi_{\mu}=\sum_{\nu} d_{\nu \mu}^{1}(-\theta) e^{i \nu \tau} \chi_{\nu}^{\prime}
$$

where $\tau=p t / \hbar, \chi_{\nu}^{\prime}$ is defined in the $Z^{\prime}$-frame, and $d_{\nu \mu}^{1}(-\theta)$ is an element of the well-known rotation matrix. Since the observation is made in the $Z$-frame, Eq. (11) is rewritten as

$$
\xi_{\mu}(t)=\sum_{\lambda} M_{\mu \lambda}(t) \chi_{\lambda}
$$

where

$$
M_{\mu \lambda}(t)=\sum_{\nu} d_{\nu \mu}^{1}(-\theta) e^{i \nu \tau} d_{\lambda \nu}^{1}(\theta)
$$

It is obvious that $\left|M_{\mu \lambda}(t)\right|^{2} \equiv P_{\lambda}^{\mu}(t)$ is the probability that an atom in $\mu$ initially would be in $\lambda$ at $t$ due to the inclined $B$. The evolution appearing as a variation of $P_{\lambda}^{\mu}(t)$ is strictly periodic with the period $\tau=2 \pi$ or $t=h / p \equiv t_{p}$. For an example, when $B=1 m G, t_{p}=$ $1.41 \mathrm{~ms}$.

It is obvious that $\left\{M_{\mu \lambda}\right\}$ is a unitary matrix and is symmetric. Due to the symmetry of $d_{\nu \mu}^{1}$, we have

$$
M_{\mu \lambda}=M_{\lambda \mu}=(-1)^{\mu+\lambda} M_{-\mu,-\lambda}^{*}
$$


In particular,

$$
\begin{gathered}
M_{1,1}=\frac{1+\cos ^{2} \theta}{2} \cos (\tau)+\frac{\sin ^{2} \theta}{2}+i \cos \theta \sin (\tau) \\
M_{1,0}=\frac{\cos \theta \sin \theta}{\sqrt{2}}(\cos (\tau)-1)+i \frac{\sin \theta}{\sqrt{2}} \sin (\tau) \\
M_{1,-1}=\frac{\sin ^{2} \theta}{2}(\cos (\tau)-1) \\
M_{0,0}=\sin ^{2} \theta \cos (\tau)+\cos ^{2} \theta
\end{gathered}
$$

The other elements of $\left\{M_{\mu \lambda}\right\}$ can be obtained via Eq. (4). Thus the evolution is completely clear.

Incidentally, based on the mean field theory, the effect of an inclined magnetic field has been studied by $\mathrm{Pu}$, et al[21]. When the atom-atom interaction and the quadratic Zeeman term have been neglected, they have derived a set of dynamic equations for the time evolution of the field amplitudes $a_{\lambda}(t)$. When the condensate is initially fully polarized $\left(a_{1}(0)=1\right.$ and $\left.a_{0}(0)=a_{-1}(0)=0\right)$, the set $a_{\lambda}(t)$ has an analytical solution as shown by Eq. (5) of their paper. It turns out $a_{\lambda}(t)=M_{1, \lambda}$. Thus, for this case, the mean field theory for many-body systems and the above simple consideration for a single-body system lead to the same result.

\section{MANY-BODY SYSTEMS WITHOUT ATOM-ATOM INTERACTION}

The initial state is assumed to be a Fock-state defined in the Z-frame as $|I\rangle \equiv\left|N_{1}^{I}, N_{0}^{I}, N_{-1}^{I}\right\rangle$, where $N_{\mu}^{I}$ is the number of atoms in $\chi_{\mu}$ initially. When the atom-atom interaction is neglected, it is straight forward to obtain the probability of an atom in $\lambda$ as

$$
P_{\lambda}^{I, \theta}(t)=\sum_{\nu}\left|M_{\nu \lambda}\right|^{2} N_{\nu}^{I} / N
$$

As before, the observation is made in the $Z$-frame. A notable point is that $P_{\lambda}^{I, \theta}$ does not depend on $N$ but the ratio $N_{\nu} / N$, and it has the same period $h / p$ as the single atom has. Furthermore, it is invariant under a reflection against the $X-Y$ plane, $P_{\lambda}^{I, \theta}=P_{\lambda}^{I, \pi-\theta}$. In particular, for $\lambda=0$

$$
P_{0}^{I, \theta}=X\left(1-3 \frac{N_{0}^{I}}{N}\right)+\frac{N_{0}^{I}}{N}
$$

where

$$
X=\frac{\sin ^{2} \theta}{2}\left\{1-\sin ^{2} \theta \cos ^{2}(\tau)+\cos ^{2} \theta[1-2 \cos (\tau)]\right\}
$$

Eq. (10) implies that the probability is not at all affected by the initial magnetization $N_{1}^{I}-N_{-1}^{I} \equiv M^{I}$, but is seriously affected by the number of atoms initially in $\mu=0$. From Eq. (10), we know that whether $N_{0}^{I} / N$ is larger or smaller than $1 / 3$ is crucial to the evolution. If $N_{0}^{I}=N / 3, P_{0}^{I, \theta}$ would remain constant (without evolution). Otherwise, $P_{0}^{I, \theta}$ will oscillate around a background, and the amplitude would become the largest if $N_{0}^{I}=N$. On the other hand, if $Z^{\prime}$ and $Z$ overlaps, we have $X=0$ and $P_{0}^{I, \theta}$ remains also constant as expected.

The time-dependent magnetization $N\left(P_{1}^{I, \theta}-P_{-1}^{I, \theta}\right) \equiv$ $N P_{\text {mag }}^{I, \theta}$ can be obtained from Eq. (9), we have

$$
P_{\text {mag }}^{I, \theta}(t)=M^{I}\left[\cos ^{2} \theta+\sin ^{2} \theta \cos (\tau)\right] / N
$$

It implies that the time-dependent magnetization depends on the initial magnetization $M^{I}$ but is not affected by $N_{0}^{I} / N$. In particular, if the system is zero-polarized initially, $P_{\text {mag }}^{I, \theta}(t)$ remains zero (without evolution). On the other hand, when $Z^{\prime}$ and $Z$ overlaps, $P_{\text {mag }}^{I, 0}(t)$ remains to be a constant $M^{I} / N$ as expected.

Incidentally, when $\theta=\pi / 4$, the evolution of $P_{\lambda}^{I, \theta}(t)$ has been calculated numerically in 21] and plotted in Fig.3 of their paper. We found that the difference between their numerical results and those from Eq.(9) is very small. The small difference implies that, in the early stage of evolution, the strong oscillation shown in their figure is essentially caused by the inclined field and is less affected by the interaction.

\section{MANY-BODY SYSTEMS WITH ATOM-ATOM INTERACTION}

For realistic condensates of ${ }^{87} \mathrm{Rb}$ atoms as an example, the interaction

$$
v_{i j}=\delta\left(\mathbf{r}_{i}-\mathbf{r}_{j}\right) \sum_{s} g_{s} P_{i j}^{s}
$$

where the strength $g_{s}=4 \pi \hbar^{2} a_{s} / M, M$ is the mass of atom. $P_{i j}^{s}$ is the projection operator of the $s$-spin-channel ( $s=0$ or 2 , which is the total spin of the two atoms $i$ and $j$ ). $a_{0}=101.8 a_{B}, a_{2}=100.4 a_{B}$ from [23]. It is assumed that the number density of the condensate and the temperature are sufficiently low so that the singlespatial-mode approximation can be adopted 24]. Under this approximation, when an irrelevant constant and the quadratic Zeeman term have been dropped, the Hamiltonian reads [10]

$$
H=G \hat{S}^{2}-p \sum_{i} \hat{S}_{Z^{\prime} i}
$$

where $\hat{S}$ is the operator of the total spin of the manybody system, $G=\frac{1}{6}\left(g_{2}-g_{0}\right) \int d \mathbf{r}|\phi(\mathbf{r})|^{4}, \phi(\mathbf{r})$ is the spatial normalized wave function of an atom (all atoms are assumed to condense into this state). Since the details of $\phi(\mathbf{r})$ affects only the strength $G$, it is not essential to our 
qualitative results. Therefore, it is simply evaluated via the Thomas-Fermi approximation.

Let us introduce the total spin-state $\vartheta_{S, M}$ of the whole system with conserved total spin $S$ and its $Z$-component $M$. The overlap of this state and the initial state $\left\langle\vartheta_{S, M} \mid N_{1}^{I}, N_{0}^{I}, N_{-1}^{I}\right\rangle \equiv \delta_{M, M^{I}} D_{M^{I}, N_{0}^{I}}^{N, S}$ is essential to the following calculation. These coefficients have been given explicitly by $\mathrm{Wu}$ (Eqs. (36) and (37) of [25]). They could be derived also by a set of recursion formulae [26]. On the other hand, we define further the total spin states $\vartheta_{S, M^{\prime}}^{\prime}$ relative to the $Z^{\prime}$-frame, $\vartheta_{S, M^{\prime}}^{\prime}$ is related to $\vartheta_{S, M}$ via a rotation. Thereby the initial state defined in the $Z$-frame can be expanded by $\vartheta_{S, M^{\prime}}^{\prime}$ as

$$
|I\rangle=\sum_{S, M^{\prime}} C_{S, M^{\prime}}^{I, \theta} \vartheta_{S, M^{\prime}}^{\prime}
$$

where $C_{S, M^{\prime}}^{I, \theta}=D_{M^{I}, N_{0}^{I}}^{N, S} d_{M^{\prime}, M^{I}}^{S}(-\theta)$

Since the set $\vartheta_{S, M^{\prime}}^{\prime}$ are the eigen-states of the Hamiltonian with the eigen-energy $G S(S+1)-p M^{\prime}$, the timedependent solution of the system reads

$$
\Psi(t)=\sum_{S, M^{\prime}} C_{S, M^{\prime}}^{I, \theta} e^{-i\left(G S(S+1) / p-M^{\prime}\right) \tau} \vartheta_{S, M^{\prime}}^{\prime}
$$

By using the fractional parentage coefficients given in [27, 28], we can extract the spin-state of a single particle (say, particle 1) from $\vartheta_{S, M^{\prime}}^{\prime}$ as

$$
\begin{aligned}
\vartheta_{S, M^{\prime}}^{\prime}= & \sum_{\mu} \chi_{\mu}^{\prime}(1)\left[\mathcal{A}\left(N, S, M^{\prime}, \mu\right) \vartheta_{S+1, M^{\prime}-\mu}^{[N-1] \prime}\right. \\
& \left.+\mathcal{B}\left(N, S, M^{\prime}, \mu\right) \vartheta_{S-1, M^{\prime}-\mu}^{[N-1] \prime}\right]
\end{aligned}
$$

where

$$
\begin{gathered}
\mathcal{A}\left(N, S, M^{\prime}, \mu\right)=\left[\frac{(N-S)(S+1)}{N(2 S+1)}\right]^{1 / 2} C_{S+1, M^{\prime}-\mu, 1, \mu}^{S, M^{\prime}} \\
\mathcal{B}\left(N, S, M^{\prime}, \mu\right)=\left[\frac{S(N+S+1)}{N(2 S+1)}\right]^{1 / 2} C_{S-1, M^{\prime}-\mu, 1, \mu}^{S, M^{\prime}}
\end{gathered}
$$

where the Clebsch-Gordan coefficients have been introduced. Note that in Eqs. (18) and (19) $N-S$ must be even, otherwise the state $\vartheta_{S, M^{\prime}}^{\prime}$ does not exist.

Since the observation is made in the $Z$-frame, the single particle state $\chi_{\mu}^{\prime}(1)$ in Eq. (17) is further rewritten as $\chi_{\mu}^{\prime}=\sum_{\lambda} d_{\lambda, \mu}^{1}(\theta) \chi_{\lambda}$, where $\chi_{\lambda}$ is defined in the $Z$-frame.

With these transformations, eventually the probability of a particle in $\lambda$ can be extracted from $\Psi(t)$, and we have

$$
\begin{aligned}
& P_{\lambda}^{I, \theta}(t)=\sum_{\mu^{\prime}, \mu} d_{\lambda, \mu^{\prime}}^{1}(\theta) d_{\lambda, \mu}^{1}(\theta) \sum_{S, M^{\prime \prime}, M^{\prime}} \delta_{M^{\prime \prime}-\mu^{\prime}, M^{\prime}-\mu} \\
& \quad\left\{C _ { S , M ^ { \prime \prime } } ^ { I , \theta } C _ { S , M ^ { \prime } } ^ { I , \theta } \left[\mathcal{A}\left(N S M^{\prime \prime} \mu^{\prime}\right) \mathcal{A}\left(N S M^{\prime} \mu\right)\right.\right. \\
& \left.+\mathcal{B}\left(N S M^{\prime \prime} \mu^{\prime}\right) \mathcal{B}\left(N S M^{\prime} \mu\right)\right] \cos \left(\left(M^{\prime \prime}-M^{\prime}\right) \tau\right) \\
& +2 C_{S+2, M^{\prime \prime}}^{I, \theta} C_{S, M^{\prime}}^{I, \theta} \mathcal{B}\left(N, S+2, M^{\prime \prime} \mu^{\prime}\right) \mathcal{A}\left(N S M^{\prime} \mu\right) \\
& \left.\quad \cos \left(\left[4 G(S+3 / 2) / p-\left(M^{\prime \prime}-M^{\prime}\right)\right] \tau\right)\right\}
\end{aligned}
$$

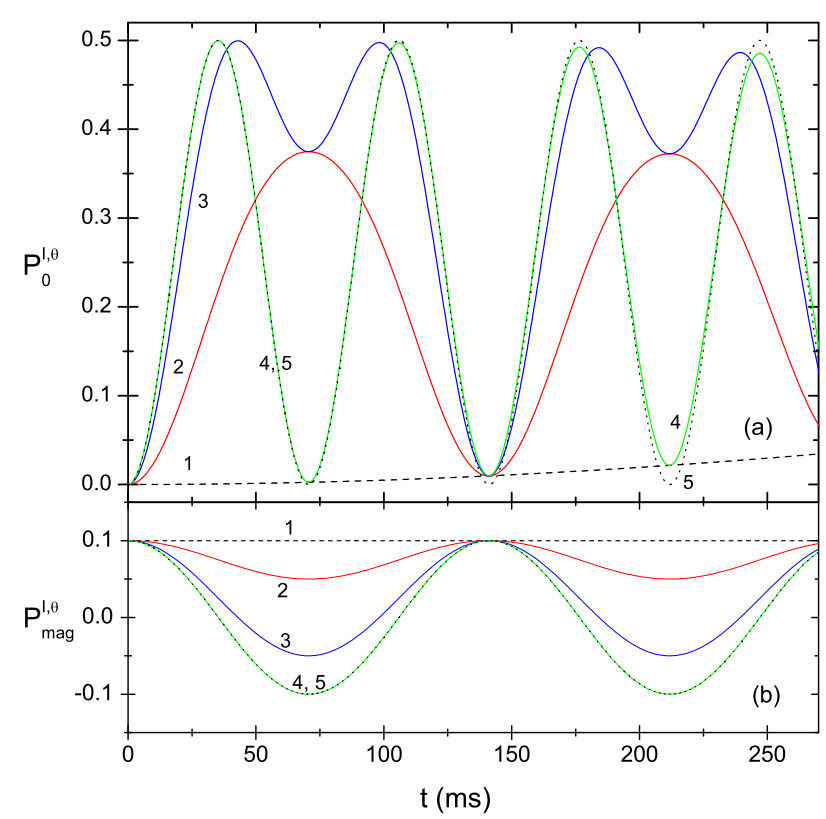

FIG. 1: (Color online) $P_{0}^{I, \theta}(t)\left(\right.$ a) and $P_{\text {mag }}^{I, \theta}(t) \equiv P_{1}^{I, \theta}-P_{-1}^{I, \theta}$ (b) against $t$ calculated from Eq. (20) with $\omega=300 \times 2 \pi$, $N=100$ and $B=0.01 m G$. The initial state has $M^{I} / N=0.1$ and $N_{0}^{I}=0$. For the curves " 1 " to " 5 ", $\theta=0, \pi / 6, \pi / 3, \pi / 2$, and $\pi / 2$, respectively. $P_{\lambda}^{I, \pi-\theta}(t)=P_{\lambda}^{I, \theta}(t)$ holds always. The curve " 1 " is identical to the one with $B=0$. " 5 " is for the case with the interaction ignored $(G=0)$.

This is a generalized version of Eq. (9) with the realistic interaction taken into account. It is also a generalization of Eq. (3) of [29], taking the effect of the inclined magnetic field into account. When $\theta=0$, Eq. (20) is identical to Eq. (3) of [29].

Eq. (20) provides an analytical description of the evolution and all the coefficients involved have analytical forms. Based on Eq. (20), numerical results are shown by the following figures as examples to demonstrate the feature of evolution. The condensate of ${ }^{87} \mathrm{Rb}$ atoms is assumed to be trapped by a harmonic potential with $\omega=300 \times 2 \pi$. A very weak field with $B=0.01 \mathrm{mG}$ is chosen. This is enough to show the effect of the linear Zeeman term. Although Eq. (20) holds for arbitrary $N$, numerical results are limited by the ability of computer. As the first example, $N=100$ is chosen.

In Figs. 1 and 2, $\theta$ is given at a set of values. Related to the figures the following points are noted.

(i) Comparing curves "2" to "4" (in solid line) with "1" (in dash line), the strong and swift oscillation caused by the inclined weak magnetic field is impressive. The evolution is highly sensitive to $\theta$.

(ii) When the interaction is ignored, the evolution is described by the curve "5" (in dotted line) which arises purely from the inclined field. Comparing " 4 " and " 5 " (both have $\theta=\pi / 2$ ), we know that the effect of interaction is weak in the early stage because the two curves almost overlap. However, if $t$ is larger, the influence of in- 


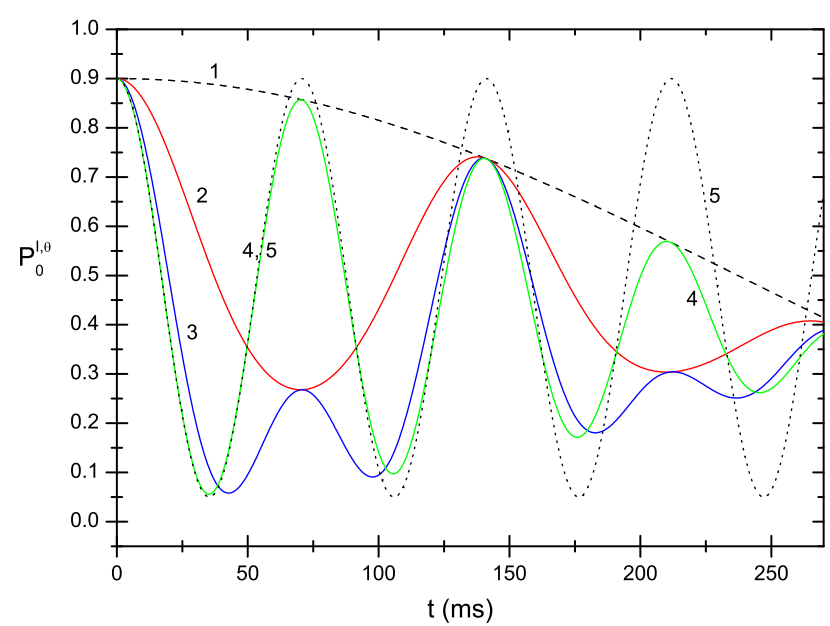

FIG. 2: (Color online) All the same as Fig. 19 but with $N_{0}^{I}=$ 0.9 .

teraction would become more and more explicit, and the deviation between " 4 " and " 5 " would be serious (refer to Fig. 2).

(iii) The evolutions shown by the curves (except " 1 ") are nearly periodic with the period $t_{p}=h / p=141 \mathrm{~ms}$. If the interaction is removed or if $G / p$ is an integer, it would be exactly periodic as shown by Eq. (20). However, since $t_{p}$ is reversely proportional to $B$, the cycles of oscillation would become very dense if $B$ is large. For instance, when $B \geq 1 m G$, the curves of $P_{\lambda}^{I, \theta}(t)$ with $\theta \neq 0$ look like a band. The width of the band is equal to a double of the amplitude of oscillation, and therefore depends on $\theta$. However, if $\theta$ is not small (say, $\theta \geq \pi / 15$ ), the width would be too broad and the band is difficult to be determined. Thus, when a stronger field is used in experiments, only the cases with small $\theta$ are meaningful.

(iv) When the field is not inclined, magnetization is conserved as shown by " 1 " of Fig. 1 b. The inclination breaks the conservation and causes oscillation as shown by " 2 " to " 4 " of Fig. 11b. The oscillation would have the largest amplitude if $\theta=\pi / 2$. Meanwhile, $P_{m a g}^{I, \pi / 2}(t)$ oscillates between $\pm M^{I} / N$. It implies that, when the initial magnetization is larger, the amplitude is larger. The overlap of " 4 " and " 5 " in 1 b implies that the interaction plays no role in the oscillation of magnetization.

(v) A larger $\omega$ would reduce the size of the system. Since the strength $G$ depends on the spatial wave function and would become larger if the size is smaller, the effect of interaction would become stronger if $\omega$ is larger.

(vi) Although the evolution caused by the inclined field does not depend on $N$ as mentioned, the evolution caused by interaction does. Since all the solid curves of Fig. 1 appear to be strictly confined by the dashed curve, how these curves vary with $N$ depends on how the dashed curve varies with $N$. The latter is referred to previous literatures [10].

(vii) When the quadratic Zeeman term is taken into account, there is no analytical solution. However, the evolution can be solved numerically. We found that, when the field is weak (say, $B \leq 0.1 m G$ ), the effect of the quadratic term is negligible.

In summary, an analytical approach beyond the mean field theory has been proposed to describe the spinevolution of small condensates. A magnetic field is applied along the $Z^{\prime}$-axis which is deviated from the $Z$-axis of quantization. Under the single-mode-approximation, exact time-dependent solution of the Hamiltonian has been obtained. A formula governing the evolution has been derived, and related numerical results have been presented. It was found that a very weak magnetic field can cause a strong, swift, and nearly periodic oscillation which is highly sensitive to the magnitude and direction of the magnetic field. The high sensitivity implies that the phenomenon might be useful for measuring the direction of a very weak field. When the magnetic field is stronger and the inclined angle is larger (say, $B$ is in the order of $m G$ and $\theta>\pi / 15$ ), the oscillation cycles will be very dense and the observation might be misunderstood as random fluctuations.

\section{Acknowledgments}

This work is supported by the NSFC under the grants 10874249 and from the project of National Basic Research Program of China (2007CB935500).
[1] D.M. Stamper-Kurn, M.R. Andrews, A.P. Chikkatur, S. Inouye, H.-J. Miesner, J. Stenger, and W. Ketterle, Phys. Rev. Lett. 80, 2027 (1998).

[2] J. Stenger, S. Inouye, D. M. Stamper-Kurn, H. -J. Miesner, A. P. Chikkatur, and W. Ketterle, Nature (London) 396, 345 (1998).

[3] T.-L. Ho, Phys. Rev. Lett. 81, 742 (1998).

[4] T. Ohmi and K. Machida, J. Phys. Soc. Jpn. 67, 1822 (1998).

[5] A. Gorlitz, T. L. Gustavson, A. E. Leanhardt, R. Low, A. P. Chikkatur, S. Gupta, S. Inouye, D. E. Pritchard, and W. Ketterle, Phys. Rev. Lett. 90, 090401 (2003).
[6] A. Griesmaier, J. Werner, S. Hensler, J. Stuhler, and T. Pfau, Phys. Rev. Lett. 94, 160401 (2005).

[7] A. Sorensen, L.-M. Duan, J.I. Cirac, and P. Zoller, Nature 409, 63 (2001).

[8] M.-S. Chang, C.D. Hamley, M.D. Barrett, J.A. Sauer, K.M. Fortier, W.Zhang, L. You, and M.S. Chapman, Phys. Rev. Lett. 92, 140403 (2004)

[9] M.-S. Chang, Q. Qin, W.X. Zhang, L. You, and M.S. Chapman, Nature Physics (London) 1, 111 (2005).

[10] C.K. Law, H. Pu, and N.P. Bigelow, Phys. Rev. Lett. 81, 5257 (1998).

[11] H. Pu, C.K. Law, S. Raghavan, J.H. Eberly, and N.P. 
Bigelow, Rhys. Rev. A. 60, 1463 (1999).

[12] L. Chang, Q. Zhai, R. Lu, and L. You, Phys. Rev. Lett. 99, 080402 (2007).

[13] S. Uchino, T. Otsuka, and M. Ueda, Phys. Rev. A 78, 023609 (2008).

[14] X. Cui, Y. Wang, and F. Zhou, Phys. Rev. A 78, 050701(R) (2008).

[15] J. Cheng, H. Jing, and Y. J. Yan, Phys. Rev. A 77, 061604(R) (2008).

[16] R. M. Bradley, J. E. Bernard, and L. D. Carr, Phys. Rev. A 77, 033622 (2008).

[17] J. Kronjäger, K. Sengstock, K. Bongs, New J. Phys. 10, 045028 (2008).

[18] T. Kuwamoto, K. Araki, T. Eno, and T. Hirano, Phys. Rev. A 69, 063604 (2004).

[19] J. Mur-Petit, M. Guilleumas, A. Polls, A. Sanpera, M. Lewenstein, K. Bongs and K. Sengstock, Phys. Rev. A 73, 013629 (2006).
[20] J. Kronjäger, C. Becker, P. Navez, K. Bongs, and K. Sengstock, Phys. Rev. Lett. 97, 110404 (2006).

[21] H. Pu, S. Raghavan, and N.P. Bigelow, Rhys. Rev. A., 61, 023602 (2000).

[22] S. Yi and H. Pu, Phys. Rev. A 73, 023602 (2006).

[23] E. G. M. van Kempen, S. J. J. M. F. Kokkelmans, D. J. Heinzen, and B. J. Verhaar, Phys. Rev. Lett. 88, 093201 (2002).

[24] W. Zhang, D.L. Zhou, M.-S. Chang, M.S. Chapman, and L. You, Phys. Rev. A 72, 013602 (2005).

[25] Y. Wu, Phys. Rev. A 54, 4534 (1996).

[26] M. Luo, C.G. Bao and Z.B. Li, J. Phys. B: At. Mol. Opt. Phys. 41, 245301 (2008).

[27] C.G. Bao, Acta Sci. Nat. Univ. Sunyatseni 43, 70 (2004).

[28] C.G. Bao and Z.B. Li, Phys. Rev. A 72, 043614 (2005).

[29] M. Luo, C.G. Bao and Z.B. Li, Phys. Rev. A 77, 043625 (2008). 